\title{
A unique insight into the MiRNA profile during genital chlamydial infection
}

\author{
Ifeyinwa Benyeogor', Tankya Simoneaux', Yuehao Wu', Stephanie Lundy ${ }^{1}$, Zenas George ${ }^{2}$, Khamia Ryans ${ }^{1}$, \\ Danielle McKeithen ${ }^{1}$, Roshan Pais ${ }^{1}$, Debra Ellerson ${ }^{2}$, W. Walter Lorenz ${ }^{4}$, Tolulope Omosun ${ }^{5}$, Winston Thompson ${ }^{6}$, \\ Francis O. Eko ${ }^{1}$, Carolyn M. Black' ${ }^{2}$ Uriel Blas-Machado ${ }^{3}$, Joseph U. Igietseme ${ }^{1,2}$, Qing He $\mathrm{H}^{1,2}$ and Yusuf Omosun ${ }^{1,2^{*}}$ (D)
}

\begin{abstract}
Background: Genital C. trachomatis infection may cause pelvic inflammatory disease (PID) that can lead to tubal factor infertility (TFI). Understanding the pathogenesis of chlamydial complications including the pathophysiological processes within the female host genital tract is important in preventing adverse pathology. MicroRNAs regulate several pathophysiological processes of infectious and non-infectious etiologies. In this study, we tested the hypothesis that the miRNA profile of single and repeat genital chlamydial infections will be different and that these differences will be time dependent. Thus, we analyzed and compared differentially expressed mice genital tract miRNAs after single and repeat chlamydia infections using a C. muridarum mouse model. Mice were sacrificed and their genital tract tissues were collected at 1, 2, 4, and 8 weeks after a single and repeat chlamydia infections. Histopathology, and miRNA sequencing were performed.
\end{abstract}

Results: Histopathology presentation showed that the oviduct and uterus of reinfected mice were more inflamed, distended and dilated compared to mice infected once. The miRNAs expression profile was different in the reproductive tissues after a reinfection, with a greater number of miRNAs expressed after reinfection. Also, the number of miRNAs expressed each week after chlamydia infection and reinfection varied, with weeks eight and one having the highest number of differentially expressed miRNAs for chlamydia infection and reinfection respectively. Ten miRNAs; mmu-miR378b, mmu-miR-204-5p, mmu-miR-151-5p, mmu-miR-142-3p, mmu-miR-128-3p, mmu-miR-335-3p, mmu-miR-195a-3p, mmu-miR-142-5p, mmu-miR-106a-5p and mmu-miR-92a-3p were common in both primary chlamydia infection and reinfection. Pathway analysis showed that, amongst other functions, the differentially regulated miRNAs control pathways involved in cellular and tissue development, disease conditions and toxicity.

Conclusions: This study provides insights into the changes in miRNA expression over time after chlamydia infection and reinfection, as well as the pathways they regulate to determine pathological outcomes. The miRNAs networks generated in our study shows that there are differences in the focus molecules involved in significant biological functions in chlamydia infection and reinfection, implying that chlamydial pathogenesis occurs differently for each type of infection and that this could be important when determining treatments regime and disease outcome. The study underscores the crucial role of host factors in chlamydia pathogenesis.

Keywords: miRNAs, Differential expression, Chlamydia infection, TFI, Chlamydial pathogenesis

\footnotetext{
* Correspondence: yomosun@msm.edu

'Department of Microbiology, Biochemistry \& Immunology, Morehouse

School of Medicine, 720 Westview Drive, S.W, Atlanta, GA 30310, USA

${ }^{2}$ Centers for Disease Control \& Prevention (CDC), Atlanta, GA 30333, USA

Full list of author information is available at the end of the article
}

(c) The Author(s). 2019 Open Access This article is distributed under the terms of the Creative Commons Attribution 4.0 International License (http://creativecommons.org/licenses/by/4.0/), which permits unrestricted use, distribution, and reproduction in any medium, provided you give appropriate credit to the original author(s) and the source, provide a link to the Creative Commons license, and indicate if changes were made. The Creative Commons Public Domain Dedication waiver (http://creativecommons.org/publicdomain/zero/1.0/) applies to the data made available in this article, unless otherwise stated. 


\section{Background}

Chlamydia infection is one of the most common bacterial sexually transmitted infection worldwide. The complications in women include endometritis, salpingitis, ectopic pregnancy, pelvic inflammatory disease (PID) and tubal factor infertility (TFI) [1, 2]. Approximately one-third of PID attributed to Chlamydia trachomatis infection occurs mainly in reproductive-age women [3]. Several clinical syndromes are associated with PID caused by Chlamydia, which during physical examinations presents as pelvic or lower abdominal pain or uterine or adnexal tenderness [4]. Increased levels of inflammatory markers such as mucin 16 , erythrocyte sedimentation rate, C-reactive protein, incidence of tubo-ovarian abscess, ectopic pregnancy, tubal infertility, Fitz-Hugh-Curtis syndrome and longer hospitalization are also associated with genital chlamydia infection in acute PID [5].

The developmental cycle of C. trachomatis alternates between the extracellular infectious elementary body and the intracellular, non-infectious reticulate body [6]. Columnar and transitional epithelial cells are the primary cells infected by Chlamydia [7]. C. trachomatis being an intracellular obligate parasite employs an array of host processes to support its developmental cycle [8]. This manipulation of host processes increases influx of innate cells, release of tissue damaging proteins and pro-inflammatory cytokines [8, 9]. The intense and chronic inflammatory response is maintained by reinfection or persistent infection in host cells with chlamydial infection ultimately leads to PID [2, 7, 8]. We have previously suggested that host inflammatory and antimicrobial immune responses to infection are important in determining Chlamydia-induced tubal pathology [10, $11]$; therefore, balancing these two factors is important in controlling genital chlamydial infections and preventing the sequelae. The initiation of the pathologic process of epithelial-mesenchyme transition in host epithelial cells has been shown to be important during chlamydial infection $[12,13]$. This process may progress to fibrosis and fertility-related epithelial dysfunction and provides the co-factor function for herpes virus (HPV)-related cervical epithelial carcinoma [13].

Proposed mechanisms for the inflammatory response and sequalae in Chlamydia infected host cells includes presence of chlamydia proteins and small non-coding RNAs (miRNAs) belonging to the host expressed during Chlamydia trachomatis infection [10, 13-18]. miRNAs are evolutionarily conserved, endogenous non-coding RNAs of approximately 22 nucleotides that play important regulatory roles in animals and plants by targeting mRNAs for degradation, cleavage, translational repression or occasional enhancement $[19,20]$. miRNAs have been reported to account for approximately one-third of mammalian gene expression by regulating the expression of genes involved in cellular differentiation, maintenance of cellular integrity, development, functions and normal metabolism, reproduction, fibrosis and oncogenesis [21]. miRNAs have been shown to be differentially expressed during ocular chlamydia infection [22, 23]; in addition, we and other authors have shown an association between genital chlamydia infection and differential expression of miRNAs [10]. Furthermore, we showed a direct relationship between the integrity of Dicer, a ribonuclease III enzyme, and key micro-RNAs in the reproductive system [10]. Immune cell type-specific miRNAs appear to regulate the effective immune response to chlamydial infection [24]. The development of PID due to chlamydial infection with virulent and attenuated phenotypes can be predicted using miRNAs [17] with the Chlamydia virulence associated with the miRNA expression profile of the host [25]. miRNAs have also been associated with regulating mitochondrial function which is necessary for C. trachomatis development [26]. These studies buttress the importance of miRNAs as important factors in host cell changes and response during chlamydial infection.

Although these findings show the importance of miRNAs expression in determining the outcome of chlamydial infections, they however, do not provide a full picture of the role miRNAs may play during chlamydial infection. For instance, they do not show the effect of Chlamydia trachomatis on miRNA expression over an extended period. The methods used to analyze genital tract miRNAs so far have not provided a detailed enumeration of all the known miRNAs associated with chlamydial infection. To address this knowledge gap, we investigated the total miRNA expression profile using deep sequencing, in mice infected and reinfected with $C$. muridarum. C. muridarum mouse models results in genital tract infections that are similar to acute genital tract $C$. trachomatis infections in women. The infection in mice can cause hydrosalphinx, fibrosis and infertility, which are common in infected women [27-29]. Here, we report the expression profile of miRNAs in the female mouse genital tracts at one, two, four and 8 weeks after chlamydia infection and reinfection.

\section{Results}

\section{Mice reinfected with $C$. muridarum have pronounced uterine pathology}

We performed histopathological examinations of the genital tract tissues from $C$. muridarum infected and reinfected mice and compared the changes. Significant findings were mainly detected in the uterus. When compared to controls, mice infected once had a number of lesions that increased with time, meaning that more lessions were formed in mice genital tracts as the infection progressed. The pathologic findings varied from eosinophilic changes in the endometrium to lymphocytic inflammation, with associated apoptotic necrosis of endometrial epithelia (Fig. 1a-f). 
Reinfected mice had an increased number of tissue alterations in the uterus and oviduct, compared to control mice. The number of lesions was not associated with time (Fig. 2a-f), but included periglandular inflammation with and without the formation of new fibrous tissue (periglandular fibroplasia) (Fig. 2b and c). The character of the periglandular inflammation was predominantly lymphocytic and plasmacytic, with formation of lymphoid follicles (Fig. 2f). In addition, there was thickening of the lining of the uterus, apoptotic necrosis, and distension of the endometrium (Fig. $3 \mathrm{~b}$ and c). The results suggested that regulated pathophysiologic processes were induced during a primary and repeat genital chlamydial infection.

\section{Pattern and number of differentially expressed miRNAs are different after a primary C. muridarum infection and reinfection and is time dependent}

We investigated the role of miRNAs in regulating the pathophysiologic processes induced during a primary and repeat genital chlamydial infection. We analyzed the miRNA sequencing data and observed that there were more miRNA differentially expressed in reinfection compared to single infection, however in both cases more miRNAs were down regulated during $C$. muridarum infection than were upregulated (Fig. 4a-g; Tables 1, 2, 3). The number of miRNAs that were differentially expressed varied based on the time after infection when the samples were collected. After a single infection, the highest number of differentially expressed miRNAs was observed in week 8 . However, after reinfection weeks 1 and 2 had the highest number of differentially expressed miRNAs (Fig. 4e and f; S. Figs. 1 and 2; Table 1). To eliminate any chance of having false positives in the miRNA analysis, we performed a false discovery rate (FDR) analysis. This analysis reduced the number of miRNAs that were significantly differentially expressed. In all, 21 miRNAs in total were significantly expressed after a single infection, most of these miRNAs have human homologs The log fold changes ranged from -4.83 to 2.93 . Some miRNAs appeared more than once (mmu-miR-378b and mmu-miR-151-5p). miR-3470b was the most upregulated miRNA, while mmu-miR-378b was the most down regulated miRNA. Interestingly, mmu-miR-378b was upregulated in week 1 after infection and then downregulated in week 8 (Fig. 4e; Table 2). After FDR corrected analysis we obsevered that 66 miRNAs were significantly expressed during chlamydia reinfection, most of these miRNAs have human homologs. Six of the miRNAs; mmu-miR-378b, mmu-miR-106a, mmu-miR-142-3p, mmu-miR-142-5p, mmu-miR-20b-5p, mmu-miR-146a-5p; appeared more than once. Log fold changes ranged from -5.14 to 3.19 (Fig. 4f; Table 3). mmu-miR-378b was the most upregulated miRNA, while

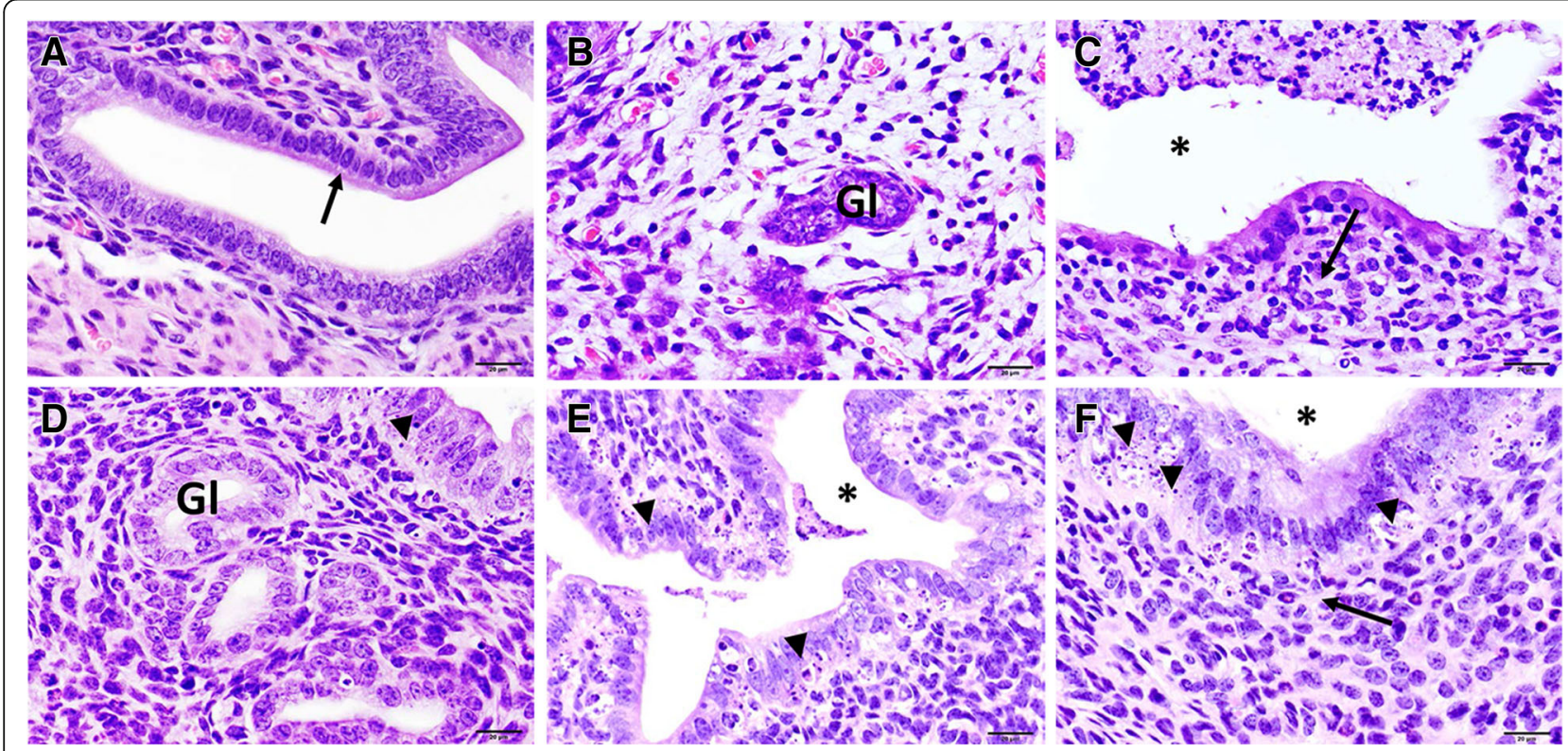

Fig. 1 Histopathological assessment of genital tract of mice infected once with Chlamydia. a. Representative image of the endometrium from an uninfected mouse. A single layer of columnar epithelia (arrow), with basally located nuclei lining the endometrium. The underlying lamina propria has scattered spindled cells, some capillaries, and occasional leukocytes. Hematoxylin and eosin (HE) stain. Scale bar $=20 \mu \mathrm{m}$. $\mathbf{b}$ and $\mathbf{c}$. Representative image of uterine endometrium with increased presence of eosinophils after the first and second weeks of infection. The lamina propria is mildly hypercellular, with loosely arranged spindled stromal cells and increased numbers of eosinophils around an endometrial gland (Gl). HE stain. Scale bar $20 \mu \mathrm{m}$. d-f. Representative images of inflammed uterine endometrium with necrosis after 4 weeks of infection. $\mathbf{d}$. The uterine lumen (asterisks) is filled with necrotic cellular debris. The mucosa has widespread epithelial apoptotic necrosis (arrowheads). The lamina propria is moderately hypercellular, with increased numbers of neutrophils, eosinophils, and few lymphocytes (arrows). HE stain. Scale bar $20 \mu \mathrm{m}$ 

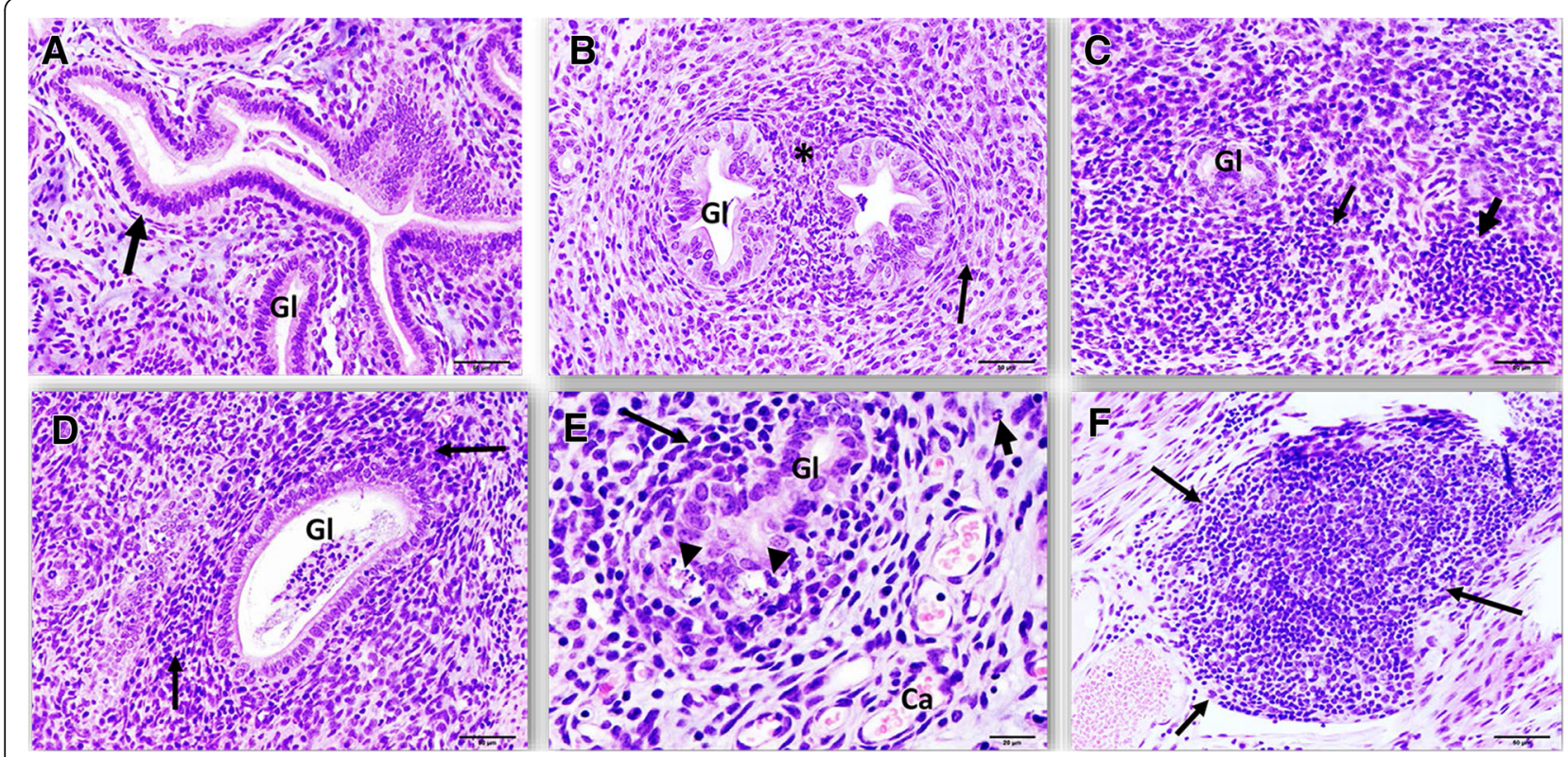

Fig. 2 Histopathological assessment of genital tract of mice infected twice with Chlamydia. a. Representative image of the endometrium from an uninfected mouse. A single layer of columnar epithelia (arrow), with basally located nuclei lining the endometrium. The underlying lamina propria has scattered spindled cells, some capillaries, and occasional leukocytes in a pale basophilic myxomatous matrix. (Endometrial gland =Gl). HE stain. Scale bar $50 \mu \mathrm{m}$. b. Representative image of uterine endometrium with periglandular fibrosis after 1 week of reinfection. Increased numbers of neutrophils (asterisk) and spindled cells (fibroblasts, arrow) are arranged around endometrial glands (Gl). HE stain. Scale bar $50 \mu \mathrm{m}$. c

Representative image of uterine endometrium with increased lymphocyte presence after 2 weeks of reinfection. Large numbers of lymphocytes (arrows) obscure and expand the tissues around an endometrial gland (Gl). HE stain. Scale bar $50 \mu \mathrm{m}$. $\mathbf{d}$ and $\mathbf{e}$. Representative image of uterine endometrium with increased lymphocyte presence after 4 weeks of reinfection with lymphocytic endometritis. Large numbers of lymphocytes, with fewer plasma cells (long arrows) and occasional neutrophils (short arrow) obscure and expand the tissues around an endometrial gland (Gl). Arrowheads point to foci of glandular apoptotic necrosis. Capillaries = Ca. HE stain. Scale bars 50 and $20 \mu \mathrm{m}$, respectively. f. Representative image of uterine endometrium with increased lymphocyte presence and lymphoid follicle formation (arrows) after 8 weeks of reinfection. An expansive lymphoid follicle obscures and expands the tissues of the deep stratum spongiosum and adjacent myometrium. HE stain. Scale bar $50 \mu m$

mmu-miR-205-5p was the most down regulated miRNA (Table 3). Similar to what we observed after a single infection, mmu-miR-378b fold change value decreased as the infection progressed from week 1 to week 8 . Ten miRNAs were found to be common in chlamydia infection and reinfection. These microRNAs are mmu-miR-378b, mmu-miR-204-5p, mmu-miR-151-5p, mmu-miR-142-3p, mmu-miR-128-3p, mmu-miR-335-3p, mmu-miR-195a-3p, mmu-miR-142-5p, mmu-miR-106a-5p and mmu-miR-92a-3p (Fig. 4g). The results suggested that these changes in miRNAs
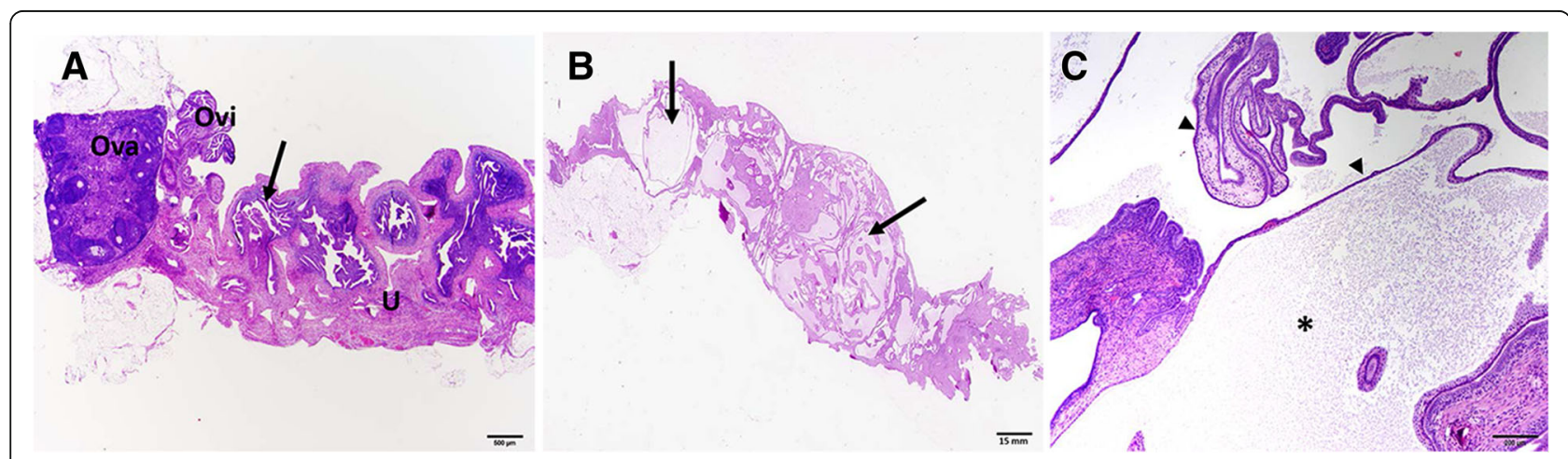

Fig. 3 Further Histopathological assessment of genital tract of mice infected twice with Chlamydia. a. Representative, longitudinal image of an uninfected mouse uterus (U), oviduct (Ovi), and ovary (Ova). A single layer of columnar epithelia (arrow), with basally located nuclei lining the endometrium (arrow). HE stain. Scale bar $500 \mu \mathrm{m}$. b and $\mathbf{c}$. Representative, sub-gross (b) and close up (c) images of a mouse uterus with marked cystic endometrial hyperplasia (arrows) 8 weeks after reinfection. Branched, papillary projections (arrowheads) of endometrium extend into the cystically dilated uterine lumen (asterisk). HE stain. Scale bars are $15 \mathrm{~mm}$ and $200 \mu \mathrm{m}$, respectively 


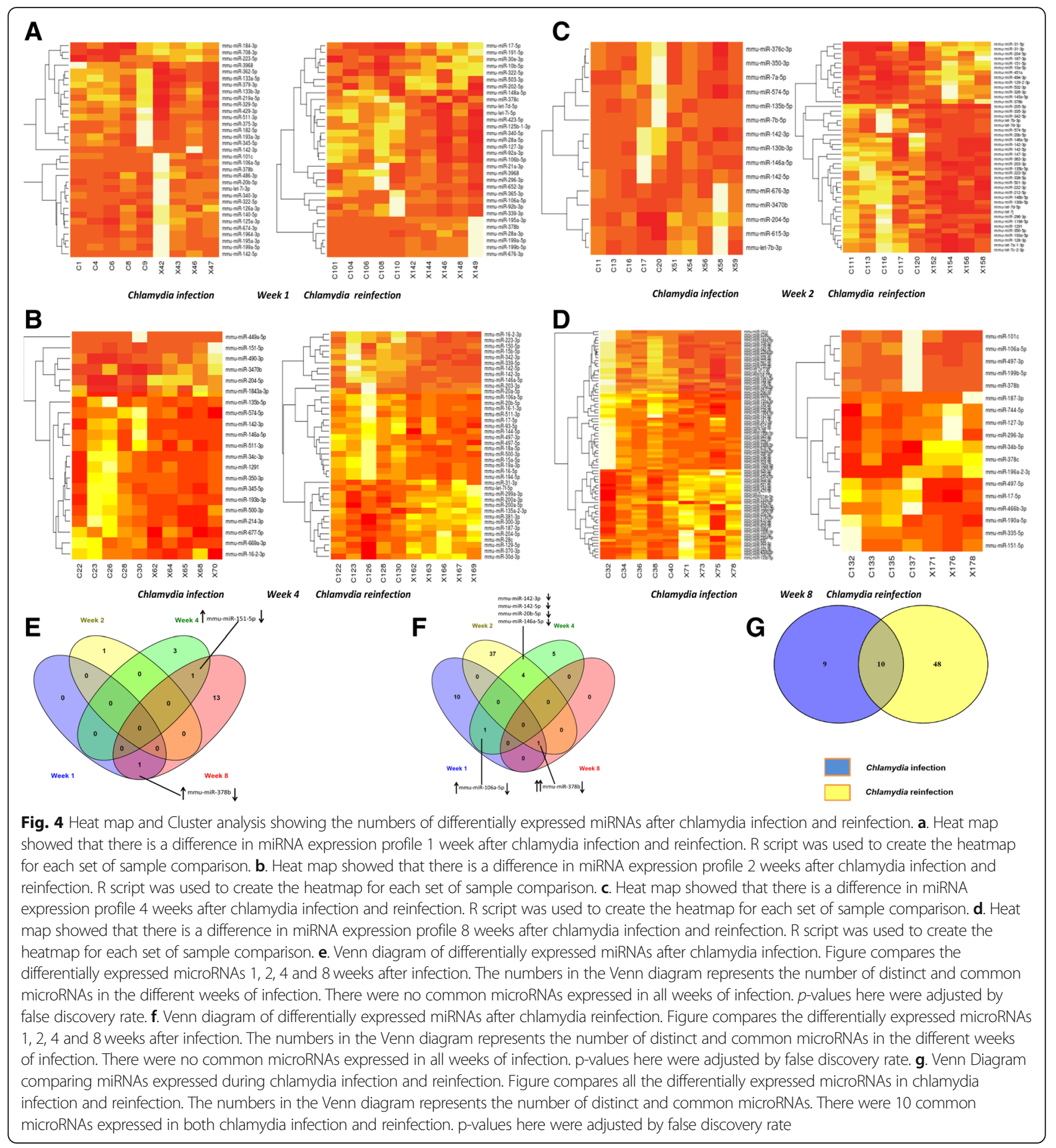

might be associated with the pathological changes that we had observed in the primary $C$. muridarum infection and reinfection, there is a need to understand what pathways they control.

\section{Differentially expressed miRNAs in C. muridarum infection regulate myriad pathways involved in disease and biological functions}

Core analysis was performed on all miRNAs expressed after single $C$. muridarum infection and reinfection in the context of molecular network and biological processes in mice using the Diana-mirPath and Ingenuity Pathway Analysis (IPA) algorithms. Using Diana-mirPath [30], our analysis showed that for the single infection, 57 pathways were predicted to be regulated by the differentially expressed miRNAs (Additional file 1: Figure S3). The pathways included extracellular matrix-receptor interaction, leukocyte trans-endothelial migration, endometrial cancer, bacterial invasion of epithelial cells, 
Table 1 Number of differentially expressed miRNAs in chlamydia infection and reinfection

\begin{tabular}{|c|c|c|c|c|c|}
\hline & Week 1 & Week 2 & Week 4 & Week 8 & Total \\
\hline Chlamydia infection $P<0.05$ & 1 & 1 & 4 & 15 & 21 \\
\hline Up regulation & 1 & 1 & 2 & 7 & 11 \\
\hline Down regulation & 0 & 0 & 2 & 8 & 10 \\
\hline Chlamydia reinfection $P<0.05$ & 12 & 42 & 10 & 1 & 65 \\
\hline Up regulation & 7 & 13 & 0 & 0 & 20 \\
\hline Down regulation & 5 & 29 & 10 & 1 & 45 \\
\hline
\end{tabular}

miRNAs expressed weeks 1, 2, 4 and 8 after infection. P-values were adjusted or controlled by false discovery rate. Upregulation indicates positive fold change of the expressed miRNAs while downregulation indicates negative fold change of the expressed miRNAs

PI3-AKT signaling, mTor signaling, protein processing in the endoplasmic reticulum, and focal adhesion. 61 pathways were predicted to be regulated by the differentially expressed miRNAs after chlamydia reinfection (Additional file 1: Figure S4). The pathways included T and B cell signaling, TGF-beta signaling, glioma, different forms of cancer, adherens junction, endocytosis, RNA degradation, bacterial invasion of epithelial cells,

Table 2 miRNAs differentially expressed after Chlamydia infection

\begin{tabular}{|c|c|c|c|c|}
\hline Week & $\operatorname{miR}$ & Fold Change(Log) & $P$ value* & Direction \\
\hline Week 1 & mmu-miR-378b & 2.91 & 0.000057 & UP \\
\hline Week 2 & $m m u-m i R-3470 b^{c}$ & 2.93 & 0.000003 & UP \\
\hline \multirow[t]{4}{*}{ Week 4} & mmu-miR-204-5p $p^{a}$ & 1.43 & 0.000276 & UP \\
\hline & $m m u-m i R-151-5 p^{b}$ & 1.83 & 0.000292 & UP \\
\hline & mmu-miR-142-3p & -1.48 & 0.000446 & DOWN \\
\hline & mmu-miR-449a-5pa & -2.07 & 0.000493 & DOWN \\
\hline \multirow[t]{15}{*}{ Week 8} & mmu-miR-378b ${ }^{a}$ & -4.83 & 0.000000 & DOWN \\
\hline & mmu-miR-128-3p & -2.01 & 0.000016 & DOWN \\
\hline & mmu-miR-151-5 $p^{b}$ & -1.78 & 0.000018 & DOWN \\
\hline & mmu-miR-101 $c^{b}$ & -3.20 & 0.000035 & DOWN \\
\hline & $m m u-m i R-335-3 p^{a}$ & 2.03 & 0.000101 & UP \\
\hline & $m m u-m i R-450 b-5 p^{b}$ & 2.11 & 0.000108 & UP \\
\hline & mmu-miR-195a-3p ${ }^{b}$ & -2.11 & 0.000222 & DOWN \\
\hline & mmu-miR-92b-3pa & 1.94 & 0.000260 & UP \\
\hline & mmu-miR-142-5p & -1.92 & 0.000319 & DOWN \\
\hline & $m m u-m i R-106 a-5 p^{a}$ & -2.14 & 0.000438 & DOWN \\
\hline & mmu-miR-690 & 1.67 & 0.000692 & UP \\
\hline & mmu-miR-148a-3pa & -1.36 & 0.001044 & DOWN \\
\hline & $m m u-m i R-450 a-5 p^{a}$ & 2.19 & 0.001104 & UP \\
\hline & $m m u-m i R-133 b-3 p^{b}$ & 1.63 & 0.001430 & UP \\
\hline & mmu-miR-92a-3p & 1.75 & 0.001695 & UP \\
\hline
\end{tabular}

miRNAs expressed weeks 1, 2, 4 and 8 after infection; ${ }^{a}$ miRNAs that have an exact human homolog in miRBase; ${ }^{b}$ miRNAs that do not have an exact human homolog in miRBase, but might be different by a few nucleotides, ${ }^{c}$ no known human homolog in miRBase. *P-value significance threshold was set at $p<0.05$. $p$-values here were adjusted or controlled by false discovery rate. Positive fold change (log) value indicates upregulation of the expressed miRNA while a negative fold change (log) value indicates downregulation of the expressed miRNA endometrial cancer, $\mathrm{FC} \psi$ receptor $(\mathrm{FC} \psi \mathrm{R})$-mediated phagocytosis, Wnt signaling and extracellular matrix (ECM) receptor interactions. More pathways were predicted to be regulated after reinfection due to the higher number of differentially expressed miRNAs, we further analysed our data using IPA, to determine if there were any subtle differences in these pathways in mice.

The IPA core expression analysis provides a rapid assessment of the signaling and metabolic pathways, upstream regulators, molecular interaction networks, and diseases and biological functions that are most likely to be perturbed based on the changes of expression of miRNAs after a single chlamydia infection and reinfection. We focused on four categories when assessing the effects of $C$. muridarum on the differential expression on miRNAs; physiological system development, diseases and disorders, molecular and cellular functions, and toxicity functions. In pathways associated with physiological system development, the results show that there were differences in pathways regulating organ development, organismal development and connective tissue development \& function after chlamydia infection and reinfection (Fig. 5a). In diseases and disorders associated pathways, there were differences in pathways regulating tumorigenesis or cancer, organismal injury \& abnormalities disease and reproductive system disease after chlamydia infection and reinfection (Fig. 5b). In pathways associated with molecular and cellular functions the results show that there were differences after a single chlamydia infection and reinfection especially in cellular development, cell cycle, and cellular growth and proliferation. (Fig. 5c).

During a single C. muridarum infection, the most significant pathways within the physiological systems development and functions category included organismal development and connective tissue development and function (Fig. 5a; Additional file 1: Table S1). The most significant pathways within the diseases and disorders category included cancer, organismal injury and abnormalities disease and reproductive system disease, these pathways had the same number [12] of miRNAs (Fig. 5b; Additional file 1: Table S1). The most significant pathways within the molecular and cellular functions 
Table 3 Differentially expressed miRNAs after Chlamydia reinfections

\begin{tabular}{|c|c|c|c|c|}
\hline Week & miR & $\begin{array}{l}\text { Fold } \\
\text { Change(Log) }\end{array}$ & $P$ value* & Direction \\
\hline \multirow[t]{12}{*}{ Week 1} & mmu-miR-378b & 3.19 & 0.0000009 & UP \\
\hline & mmu-miR-3968 & -1.75 & 0.000042 & DOWN \\
\hline & $m m u-m i R-106 a-5 p^{a}$ & 1.86 & 0.000091 & UP \\
\hline & mmu-miR-423-5p $p^{a}$ & -1.52 & 0.00016 & DOWN \\
\hline & mmu-miR-92a-3pa & -1.70 & 0.00022 & DOWN \\
\hline & mmu-miR-199a-5pa & 1.78 & 0.000257 & UP \\
\hline & mmu-miR-127-3p $p^{a}$ & -1.35 & 0.000332 & DOWN \\
\hline & $m m u-m i R-17-5 p^{a}$ & 1.20 & 0.000495 & UP \\
\hline & mmu-miR-340-5 $p^{a}$ & -1.26 & 0.000698 & DOWN \\
\hline & mmu-miR-195a-3p & 1.25 & 0.001594 & UP \\
\hline & $m m u-m i R-322-5 p^{c}$ & 1.30 & 0.00161 & UP \\
\hline & $m m u-m i R-199 b-5 p^{a}$ & 1.47 & 0.001765 & UP \\
\hline \multirow[t]{31}{*}{ Week 2} & mmu-miR-205-5 $p^{a}$ & -5.14 & 0.0000000 & DOWN \\
\hline & mmu-miR-342-5 $p^{a}$ & -3.18 & 0.0000000 & DOWN \\
\hline & mmu-miR-147-3p & -3.29 & 0.0000000 & DOWN \\
\hline & mmu-miR-135b-5 $p^{a}$ & -2.06 & 0.0000000 & DOWN \\
\hline & mmu-miR-363-3p & -2.55 & 0.000001 & DOWN \\
\hline & mmu-miR-142-3p & -2.46 & 0.000001 & DOWN \\
\hline & mmu-miR-145a-5p $p^{b}$ & 2.03 & 0.000005 & UP \\
\hline & mmu-miR-142-5 $p^{a}$ & -1.81 & 0.000012 & DOWN \\
\hline & mmu-miR-129-2-3p & 1.74 & 0.000013 & UP \\
\hline & mmu-miR-187-3p & 1.50 & 0.000039 & UP \\
\hline & mmu-miR-451a & 1.43 & 0.000105 & UP \\
\hline & $m m u-m i R-31-3 p^{a}$ & 2.07 & 0.00013 & UP \\
\hline & $m m u-m i R-326-3 p^{a}$ & 1.48 & 0.000185 & UP \\
\hline & mmu-miR-204-5p & 1.41 & 0.000188 & UP \\
\hline & mmu-miR-532-3p & 1.54 & 0.000195 & UP \\
\hline & $m m u-m i R-146 b-5 p^{b}$ & -1.34 & 0.000219 & DOWN \\
\hline & mmu-miR-146a-5 $p^{a}$ & -1.74 & 0.000231 & DOWN \\
\hline & $m m u-m i R-20 b-5 p^{a}$ & -1.73 & 0.000402 & DOWN \\
\hline & $m m u-m i R-151-5 p^{b}$ & 1.24 & 0.000438 & UP \\
\hline & mmu-miR-335-3p & -1.96 & 0.000541 & DOWN \\
\hline & $m m u-m i R-212-5 p^{a}$ & -1.18 & 0.000733 & DOWN \\
\hline & $m m u-m i R-31-5 p^{b}$ & 1.45 & 0.000829 & UP \\
\hline & $m m u-m i R-130 b-5 p^{a}$ & -1.25 & 0.000892 & DOWN \\
\hline & mmu-let-7d-3p $p^{a}$ & -1.32 & 0.001096 & DOWN \\
\hline & mmu-let-7b-3p $p^{a}$ & -1.46 & 0.001203 & DOWN \\
\hline & $m m u-m i R-378 b^{a}$ & 1.77 & 0.001282 & UP \\
\hline & $m m u-m i R-350-5 p^{c}$ & -1.33 & 0.00141 & DOWN \\
\hline & $m m u-m i R-1198-5 p^{c}$ & -1.28 & 0.001676 & DOWN \\
\hline & mmu-let-7j ${ }^{b}$ & -1.24 & 0.00175 & DOWN \\
\hline & mmu-miR-222-3p ${ }^{a}$ & -1.01 & 0.001753 & DOWN \\
\hline & mmu-miR-574-5 $p^{a}$ & -1.64 & 0.001819 & DOWN \\
\hline
\end{tabular}

Table 3 Differentially expressed miRNAs after Chlamydia reinfections (Continued)

\begin{tabular}{|c|c|c|c|c|}
\hline Week & $\mathrm{miR}$ & $\begin{array}{l}\text { Fold } \\
\text { Change(Log) }\end{array}$ & $P$ value* & Direction \\
\hline & $m m u-m i R-128-3 p^{b}$ & -1.05 & 0.00218 & DOWN \\
\hline & mmu-let-7a-1-3pa & -1.06 & 0.002268 & DOWN \\
\hline & mmu-let-7c-2-3pa & -1.06 & 0.002284 & DOWN \\
\hline & mmu-miR-203-3p & -0.98 & 0.003381 & DOWN \\
\hline & mmu-miR-1291 ${ }^{a}$ & -1.11 & 0.00341 & DOWN \\
\hline & mmu-miR-10a-5p & 0.96 & 0.003667 & UP \\
\hline & mmu-miR-338-5 $p^{a}$ & -1.14 & 0.004249 & DOWN \\
\hline & mmu-miR-223-5pa & -1.22 & 0.004819 & DOWN \\
\hline & mmu-miR-193a-5p & -1.10 & 0.005228 & DOWN \\
\hline & mmu-miR-494-3p & 1.04 & 0.005263 & UP \\
\hline & mmu-miR-296-3pa & -1.16 & 0.005263 & DOWN \\
\hline \multirow[t]{10}{*}{ Week 4} & mmu-miR-142-3pa & -2.17 & 0.0000000 & DOWN \\
\hline & mmu-miR-106a-5 $p^{a}$ & -1.77 & 0.000001 & DOWN \\
\hline & mmu-miR-142-5pa & -1.42 & 0.000048 & DOWN \\
\hline & mmu-miR-339-5p & -1.20 & 0.000052 & DOWN \\
\hline & $m m u-m i R-20 b-5 p^{a}$ & -1.42 & 0.000055 & DOWN \\
\hline & mmu-miR-146a-5p $p^{a}$ & -1.49 & 0.000209 & DOWN \\
\hline & mmu-miR-511-3pa & -1.38 & 0.000261 & DOWN \\
\hline & mmu-miR-16-2-3pa & -1.34 & 0.000433 & DOWN \\
\hline & mmu-miR-15a-5p & -1.07 & 0.000485 & DOWN \\
\hline & $m m u-m i R-497-3 p^{a}$ & -1.20 & 0.001221 & DOWN \\
\hline Week 8 & $m m u-m i R-378 b^{a}$ & -3.84 & 0.000081 & DOWN \\
\hline
\end{tabular}

miRNAs expressed weeks 1, 2, 4 and 8 after infection; ${ }^{a}$ miRNAs that have an exact human homolog in miRBase; ${ }^{b}$ miRNAs that do not have an exact human homolog in miRBase, but might be different by a few nucleotides, ${ }^{c}$ no known human homolog in miRBase. *P-value significance threshold was set at $p<0.05$. $p$-values here were adjusted or controlled by false discovery rate. Positive fold change (log) value indicates upregulation of the expressed miRNA while a negative fold change (log) value indicates downregulation of the expressed miRNAs

included cellular development, cell cycle and cell-to-cell signaling and interaction (Fig. 5 c; Additional file 1: Table S1).

During C. muridarum reinfection, the most significant pathways within the physiological system development and functions category included organ and connective tissue development (Fig. 5a; Additional file 1: Table S1). The most significant pathways within the diseases and disorders category included cancer, organismal injury and abnormalities disease (Fig. 5b; Additional file 1: Table S1). The most significant pathways within the molecular and cellular functions included, cellular development and cellular growth and proliferation (Fig. 5c; Additional file 1: Table S1). We did a further analysis of the regulated pathways and showed precisely the key steps where these miRNAs associated with chlamydia reinfection had been described to play a role (Additional file 1: Table S2). 


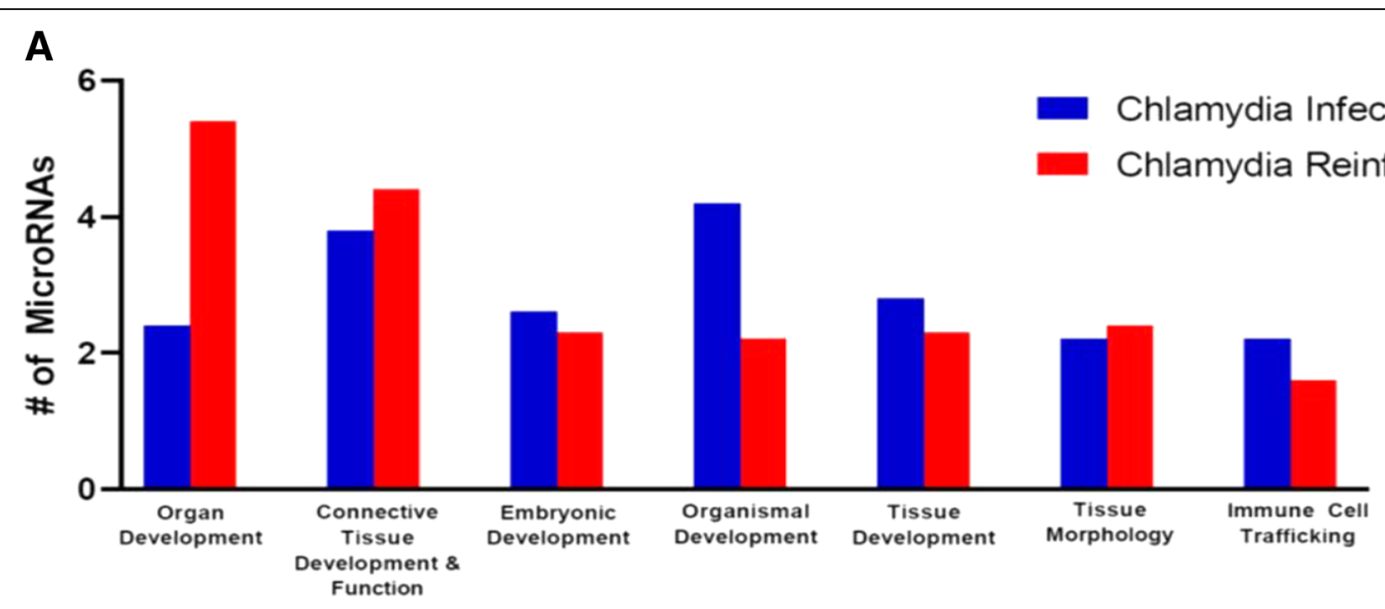

Physiological Systems Development \& Function

B

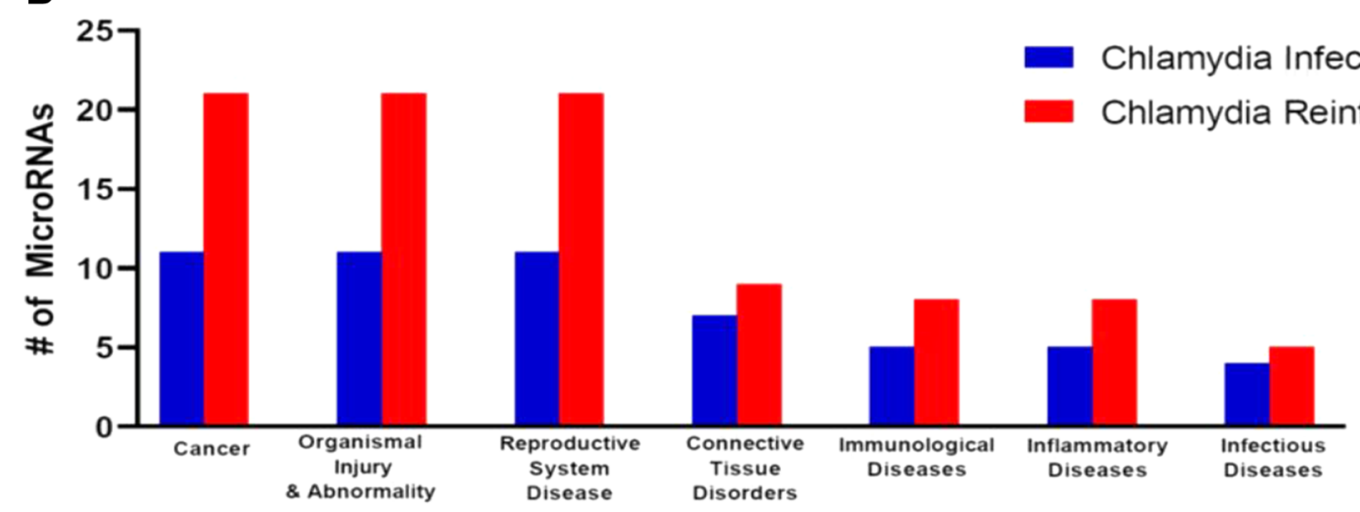

Diseases \& Disorders

C
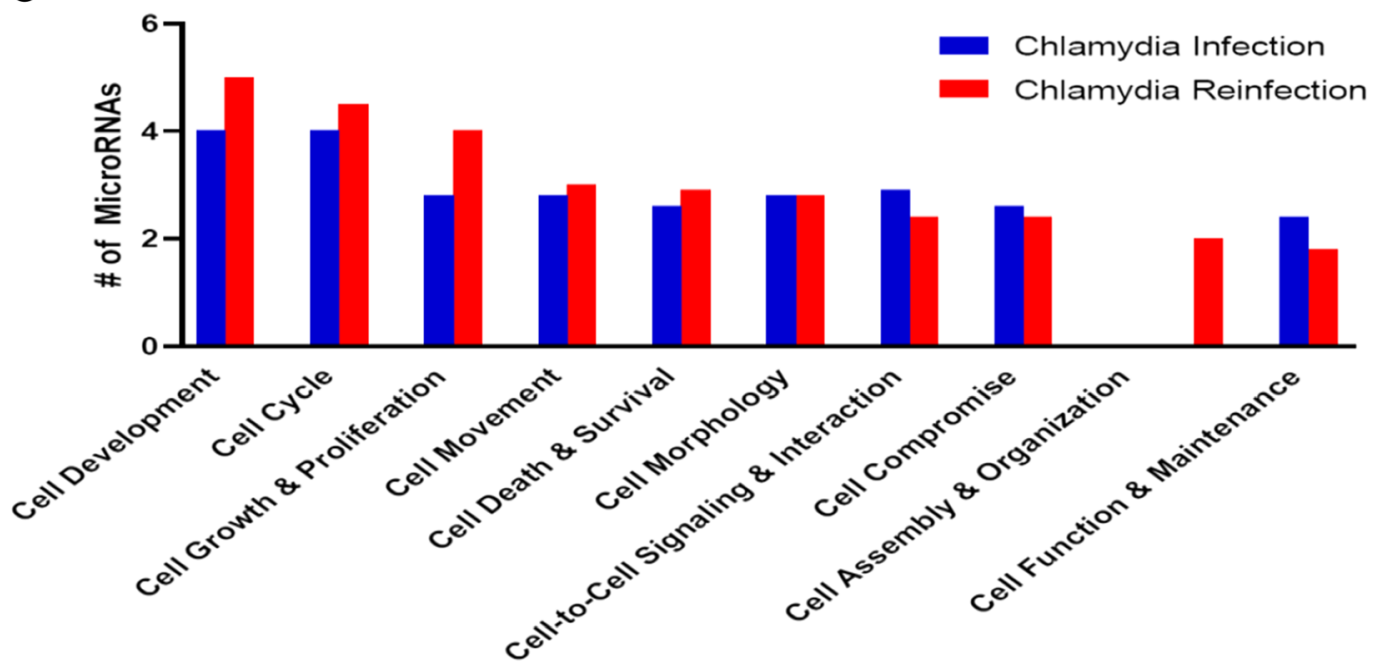

Molecular \& Cellular Function

Fig. 5 (See legend on next page.) 
(See figure on previous page.)

Fig. 5 Pathways predicted to be regulated by miRNAs differentially expressed in mice after chlamydia infection and reinfection. a. Pathways predicted to regulate physiological system development after chlamydia infection and reinfection. This analysis was determined using Ingenuity pathway analysis software (IPA). miRNAs used in the analysis were from the list derived after FDR correction. $\mathbf{b}$. Pathways predicted to regulate diseases and disorders after chlamydia infection and reinfection. This analysis was determined using Ingenuity pathway analysis software (IPA). miRNAs used in the analysis were from the list derived after FDR correction. c. Pathways predicted to regulate molecular and cellular functions after chlamydia infection and reinfection. This analysis was determined using Ingenuity pathway analysis (IPA). miRNAs used in the analysis were from the list derived after FDR correction

We evaluated the top networks of miRNAs expressed during C. muridarum infection and generated two associated networks using IPA knowledge base. Network 1 consists of miRNAs and molecules associated with cancer, organismal Injury and abnormalities and reproductive system disease. We observed 10 focus molecules. The transcription factor CCAAT/Enhancer Binding Protein Alpha (CEBPA), was the central focus molecule. Network 2 consists of miRNAs and molecules associated organismal injury and abnormalities, reproductive system disease and cancer. There were 6 focus molecules. The transcription factor CAMP Responsive Element Binding Protein 1 (CREB1), was the central focus molecule (Additional file 1: Figure S5A \& B).

We further evaluated the miRNAs networks expressed during $C$. muridarum reinfection and generated five associated network using IPA knowledge base (Additional file 1: Figure S5C-G; Additional file 1: Table S2). Network 1 consists of miRNAs and molecules associated with organismal injury and abnormalities, reproductive system disease and cancer. It has 11 focus molecules. The transcription factor Ras Homolog Family Member A (RHOA), was the central focus molecule. Network 2 consists of miRNAs and molecules associated organismal injury and abnormalities, reproductive system disease and cancer. It has 6 focus molecules. Mmu-miR-423-5p, was the central focus molecule (Additional file 1: Figure S5C-G, Additional file 1: Table S2). The results suggested that the miRNAs expression pattern after C. muridarum infection plays a role in the pathophysiologic processes associated with the infection.

\section{Discussion}

Understanding the role of host factors in the chlamydial pathogenesis is important in diagnosing infection associated complications and developing new treatments for them. In this study, we determined changes in miRNA expression after chlamydia infection and predicted the role miRNAs might play in disease outcome. Several studies have elucidated the role of miRNAs during chlamydia infection [10, 13, 17, 18, 22-26, 31]. These studies show that miRNAs are important factors in host cell changes and response during chlamydial infection. However, none of the studies on mice genital chlamydial infection have defined the full complement of miRNAs found within the genital tract. The current study was designed to sequence all the known miRNAs found within mice genital tract during chlamydial infection and reinfection. The results reported here are based on all known miRNAs found in the mouse genome. We determined the miRNA expression profiles at different time points after infection; from week 1 to 8 . This was done to follow miRNAs progression with time and determine if the changes in miRNAs expression will coincide with pathological changes identified in the mice genital tract.

Histopathological results showed that there was a significant difference in uterine tissue alterations between chlamydia infected and reinfected infected mice. Mice infected once with chlamydia had eosinophilic changes in the endometrium with lymphocytic inflammation associated with apoptotic necrosis, and these features increased over time. The uterus from chlamydia reinfected mice had increased tissue alterations such as formation of new fibrous tissues associated with inflammation, thickening of the lining of the uterus, apoptotic necrosis, and distension of the endometrium. However, there was no apparent association of the occurrence of these characteristics with time, although the the thickening of the endometrial lining was more prominent in the reinfection at week 8 . This indicates that after the first infection, pathological changes increased in severity during the reinfection. This is similar to the reported pathologies in women, with increase in complications associated with chlamydia infection occurring in recurrent infections [32].

Here we provide a further insight into the role played by miRNAs during genital chlamydial infection by providing miRNAs associated with disease progression. In addition, we depict the possible pathways associated with the differential expression of these miRNAs during chlamydial infection. In this study, we utilized next generation sequencing (NGS); the best method for determining the miRNAs expression. NGS has several advantages over microarray or quantitative (q) PCR-based miRNA expression profiling techniques. This includes, high sensitivity to determining miRNA levels over a wide dynamic range, ability to identify novel miRNAs and to detect miRNA expression levels in species for which complete genomes are not yet available. In addition, NGS can detect miRNAs that differ by just one nucleotide. Multiplexing of samples by tagging libraries with barcodes during library 
preparation is possible in NGS. However, NGS is expensive even though the price is decreasing [33, 34]. The sequences identified from the NGS were subsequently analyzed using the comprehensive analysis pipeline for miRNA-sequence data (CAP-miRSeq), which is a powerful and flexible tool used to process and analyze miRNA-seq data [35]. This study offers evidence that miRNAs differentially expressed during chlamydia infection and reinfection differ in number, types and regulatory pathways in a time dependent manner.

Initial cluster analysis show a difference in miRNA expression during chlamydia infection and reinfection at weeks one, two, four and eight after chlamydial infection. Other studies have looked at earlier time points (1 week to 12 days) of a single infection $[17,18,25]$ or only during a second infection [10]. Here we report that there is a difference in the miRNA signature based on the number of chlamydial infections. During single chlamydia infection, 11 miRNAs were up-regulated while 10 miRNAs were down-regulated. During the chlamydia reinfection, 20 miRNAs were up-regulated while $45 \mathrm{miR}$ NAs were down-regulated. We have shown in previous studies that more miRNAs are downregulated during genital chlamydia infection than are upregulated, and we related these changes in differential expression to increased expression of genes associated with fibrosis, epithelial to mesenchymal transition, cell proliferation, growth and differentiation [10, 36]. Gupta et al., also showed that several miRNAs are down regulated during chlamydia infection [18]. However, in ocular chlamydia infection there appears to be more upregulation of miRNAs than down regulation [23]. Our findings confirm that there is a alteration in the miRNAs profile during chlamydia infection and reinfection. In addition, we provide information on the miRNA profile after chlamydia infection and reinfection over an extended period (8 weeks). Our data shows that more miRNAs were differentially expressed in the 8 week of the first infection and the second week of reinfection. This correlated with the progression of pathological changes which we had noticed during the first infection. In the repeat infection, after the second week, we did not notice appreciable changes in pathology. This implies an association between pathology and the number of differentially expressed miRNAs at those timepoints. Selection of miRNAs based on FDR has given us an insight into the miRNAs associated with pathogenesis caused by Chlamydia. Thus we can imply that the difference in miRNA expression profile and number during chlamydia infection and reinfection could be as a result of regulation through the quick induction of adaptive and protective immunity against Chlamydia [27, 37].

Pathway analysis shows that miRNAs expressed in chlamydia infection and reinfection are involved in regulating several pathways from extracellular matrix-receptor interaction, leukocyte trans-endothelial migration, bacterial invasion of epithelial cells, phosphoinositide 3-kinase - protein kinase B (PI3-AKT) signaling, mammalian target of rapamycin (mTor) signaling, protein processing in the endoplasmic reticulum, focal adhesion, $T$ and $B$ cell signaling, Transforming growth factor beta (TGF-beta) signaling, glioma, different forms of cancer, adherens junction, endocytosis, RNA degradation, FC $\psi$ R-mediated phagocytosis and Wnt signaling. Chlamydia reinfection had more pathways because more miRNAs were differentially expressed after repeat infection. Using IPA we were able to discern differences in networks between chlamydia infection and reinfection involving the cancer, organismal Injury and abnormalities and reproductive system disease, where the networks had different focal molecules. Indicating that the pathogenesis in chlamydia infection and reinfection follow different paths.

Use of miRNAs to understand the pathogenesis of Chlamydia is important, our results have some similarities with work previously done, we observed mmu-miR-142 [23, 25]; mmu-miR-146 [18]; mmu-miR-147 [22]; mmu-miR-199b, mmu-miR-142-3p, mmu-miR-204, mmu-miR-449a-5p, mmu-miR-101, mmu-miR-340 [10]. Our previous study [10] had the closest resemblance to our present data with $5 \mathrm{miR}$ NAs being similar. We had reported miRNAs expressed after a reinfection and our present data shows that more miRNAs are differentially expressed during chlamydia reinfection. However, it should be noted that we had used Lymphogranuloma venereum instead of C. muridarum. During a single chlamydia infection, we observed two miRNAs (mmu-miR-378b and mmu-miR-151-5p) changed direction in their differential expression. miRNA-378b has been shown to be important in regulating epithelial to mesenchymal transition (EMT), keratinocyte differentiation and proliferation, fibrosis and aromatase expression in the genital tract [38-45]. miR-151-5p regulates tumor cell migration and spread; it is blocked by Rho GDP dissociation inhibitor alpha (Rho GDI alpha), and associated with the focal adhesion kinase gene $[46,47]$. It positively regulates cell apoptosis by targeting the expression of tumor necrosis factor superfamily member 10 (TNFSF10) and fibronectin type III domain containing 1 (FNDC1) in mice with premature ovarian failure [48]. The increased pathology including increased migration of eosinophils/neutrophils and lessions that increased with time after single chlamydia infection might be associated with the expression of mmu-miR-378b and mmumiR-151-5p. During chlamydia reinfection, the formation of new fibrous tissues associated with inflammation, thickening of the lining of the uterus, apoptotic necrosis, and distension of the endometrium noticed 4 weeks after infection might be associated with the down regulation of mmu-miR-378b, mmu-miR-146a-5p, mmu-miR-20b-5p, mmu-miR-142-5p, mmu -miR-142-3p and mmumiR-106a-5p. These miRNAs have been associated with 
EMT and fibrosis [49-54], which we have hypothesized and shown as being important in the damage to the genital tract during chlamydia pathogenesis $[10,13,36]$. These miRNAs are also associated with cancer [49-52, 54], recently a relationship between chlamydia and ovarian cancer was described [55]. In addition we observed that miR-204-5p, miR-151-5p, miR-378b, miR-142-3p, miR-128-3p, miR-335-3p, miR-142-5p, miR-106a-5p, miR-195a-3p and miR-92a-3p were common in both infections. miR-204 targets Janus kinase 2 (JAK2), and regulates epithelial to mesenchymal transition by targetting Specificity Protein 1 (SP1) in tubular epithelial cells; regulates protein kinase RNA-like endoplasmic reticulum kinase (PERK) and unfolded protein response [56-58], which we have shown to involved in chlamydial pathogenesis [59]; miR-106a-5p suppress the proliferation, migration and invasion of osteosarcoma cells, and is a good predictor of prognosis in hepatocellular cancer [60, 61]; miR-195a-3p targets matrix metalloproteinase 2 (MMP2) in murine mesenchymal stem cells thus inhibiting angiogenesis [62]. MMP2 expression in wild type mice oviduct increases by day 7 after chalmydia infection [63]. miR-92a-3p is significantly expressed in cervical cancer tissue cell lines thus promoting cervical cancer cell proliferation, cell cycle transition from gap 1 (G1) to synthesis (S) phase and invasion [64]. Intrestingly, it has been reported in dual RNA-Seq analyses of in vitro chlamydia infected epithelial cells, that infected epithelial cells expressed aberrant immune dampening and fibrotic responses, which included increased expression of TGF-beta, MMP2 and other ECM proteins [65]. Our results show that the miRNAs identified in this study control the expression of genes linked to chlamydial pathogenesis. Therefore, we speculate that we can use these miRNAs as indicators of chlamydial pathogeneses and maybe even a biomarker for disease leading to PID and infertility. It should be pointed out that the literature is strewn with cancer associated miRNAs and it is possible that associations with cancer noticed in this study maybe cursory.

\section{Conclusion}

The findings from our study show that chlamydial infection induces differentially expressed miRNAs that play a significant role in organismal injuries and abnormalities, cellular development, reproductive disorders, cell cycle, inflammatory response, fibrosis, cancer and connective tissue disorders. The targets and pathway analyses indicate that specific miRNAs are involved in the primary infection and reinfection, although there were overlaps. In addition, our results are corroborated by previous human research findings that have established an association between recurrent or persistent endocervical chlamydial infection in women and an increased risk of pelvic inflammatory disease and scarring sequelae in the upper genital tract, which have also been demonstrated in several animal models [2, 32]. This study highlights the need to further study the association of genital Chlamydia infection with the promotion of cancer in women, because several miRNAs in this study having been associated with cancer in other studies $[46,50,60,61,64,66]$. The highly interconnected networks of miRNAs generated from our data shows that there are differences in the focus molecules involved in significant biological functions during chlamydia infection and reinfection, implying that chlamydial pathogenesis may be distinct for each type of infection and that this could be important when determining treatments regime and disease outcome. Despite the strengths of our study, involving: a) next generation sequencing, b) a single infection and reinfection, and c) sample collection up to 8 weeks after infection, it has some limitations. We did not carry out a third infection or assess the effects of antibiotics treatment on each infection, all of which may play a role in miRNA expression and pathogenesis in human infections. Although only certain aspects of the pathogenesis of chlamydial infection in the mice model mirror the human infection [2], our findings show the importance of miRNAs regulation and expression in chlamydial infection and pathogenesis, which has implications for humans. In summary, our study findings provides substantial evidence that miRNAs regulate the host responses and biological processes that are associated with Chlamydia pathogenesis.

\section{Methods}

\section{Ethics statement}

The Institutional Animal Care and Use Committee of Morehouse School of Medicine (MSM-IACUC) approved the animal care and use protocol (\# 16-24), which we followed in this study. MSM-IACUC adheres to the Natinal Institute of Health (NIH) guidelines for the care and use of laboratory animals, with Public Health Service (PHS) policy, and the Animal Welfare Act.

\section{Chlamydia stocks}

Stocks of Chlamydia muridarum Nigg (the agent of mouse pneumonitis) used for infections were prepared by propagating elementary bodies (EBs) in McCoy or HeLa cells (Division of Scientific Research, Centers for Disease Control and Prevention, Atlanta), according to standard procedures. Chlamydia stock titers were expressed as inclusion-forming units (IFU) per milliliter [67].

\section{Animals}

Female C57BL/6 J wild type mice were purchased from Jackson Laboratory (Bar Harbor, MA), fed food and provided water ad libitum, and maintained in laminar flow racks under pathogen-free conditions with a $12 \mathrm{~h}$ light and $12 \mathrm{~h}$ dark cycle. The protocols involving mice were 
approved by MSM-IACUC. For all invasive procedures mice were anesthesized using isoflurane. Mice were placed in a chamber with $2-4 \%$ gas concentration of isoflurane in a fume hood for $30 \mathrm{~min}$. Mice were euthanized using carbon dioxide asphyxiation followed by cervical dislocation. This method of euthanasia is recommended by the Panel on Euthanasia of the American Veterinary Medical Association.

\section{Infectivity}

6-8 weeks old Female C57BL/6 [40] were infected and reinfected (interval of 4-weeks) intravaginally with $1 \times 10^{5}$ IFU /mouse of live C. muridarum approximately 7 days after administration of $2.5 \mathrm{mg} /$ mouse Depo Provera (medroxy-progesterone Acetate; Pfizer Inc., NY). Control mice [40] were sham infected with sterile PBS approximately 7 days after administration of $2.5 \mathrm{mg} /$ mouse Depo Provera. Mice were determined to be infected by periodic cervicovaginal swabbing and isolation of Chlamydia in tissue culture.

\section{Histopathology}

Histopathology of the genital tract associated with chlamydial infection was investigated. 40 mice were infected and reinfected intravaginally with C. muridarum $\left(1 \times 10^{5}\right.$ IFU per mouse) approximately 7 days after administration of $2.5 \mathrm{mg} / \mathrm{mouse}$ Depo Provera (medroxy-progesterone Acetate; Pfizer Inc., NY). The entire genital tract from the vagina to the ovary was collected from infected and control mice mice at one, two, four and 8 weeks after infection ( 5 mice per time point) and fixed in 10\% formaldehyde. The samples were embedded in paraffin, cut longitudinally into $5 \mu \mathrm{m}$ sections to include the cervix, uterine horns, oviducts and ovaries as well as the luminal structures, and stained with hematoxylin and eosin. Blinded to group designations, a board-certified pathologist (UBM) evaluated the uteri, oviducts and ovaries for the presence of lesions including, but not limited to inflammation, edema, fibrosis, and luminal distension. Lesions were recorded as either absent or present. If absent (i.e., histologically normal), a score of 0 was assigned. If present, the severity of the lesions was recorded as minimal, mild, moderate, or severe, with severity scores of 1 through 4, respectively. Lesion distribution was recorded as focal, multifocal, or diffuse, with distribution scores of 1,2 , or 3 , respectively. A total severity-and-distribution group score was calculated by adding individual distribution and severity scores. Because the group sizes were uneven, an average severity-and-distribution group score was calculated by dividing the total severity-and-distribution score by the numbers of animals in the group. Note that control and infected mice were from the same batch of mice and that each time point had its own control group.

\section{Small RNA library construction and Illumina small RNA deep sequencing}

Genital tract samples were harvested after sacrificing infected and control mice mice at one, two, four and 8 weeks after infection ( 5 mice per time point) and frozen immediately on dry ice and then transferred to a -80 freezer. For non-biased recovery of miRNA, total RNA was isolated using the Direct-zol RNA miniprep (Zymo Research, Irvine CA). RNA integrity was assessed using the Agilent Technologies 2100 Bioanalyzer. Samples were submitted to the University of Georgia Georgia Genomic and Bioinformatic Core (GGBC) for library preparation with the the TruSeq Small RNA Library Preparation Kit (Illumina, San Diego, CA). Samples were sequenced on an Illumina NextSeq 500 instrument using a SE75 run High Output flow cell (Illumina, San Diego, CA, USA), inserts used were $25-75$ bases. This assay was validated using qPCR (Additional file 1: Figure S7A \& B). Note that control and infected mice were from the same batch of mice and that each time point had its own control group.

\section{Bioinformatics analysis}

NGS data analyses were performed by the GGBC. Quality of raw sequence reads was assessed using FastQC [68] (sequencing depth $\geq 4$ million reads). The data were mapped to the $M$. musculus mm10 reference genome and to the MirDeep mouse miRNA database for identifying novel and known miRNA's, respectively, using the CAP-miRSeq pipeline (version 1.1) [35]. The pipeline leverages several software packages for adapter removal and trimming, read alignment and manipulation of sequence alignment map/binary alignment map (SAM/ BAM) and fastq/fasta files (Additional file 1: Figure S8) and implements edge $\mathrm{R}$ for differential expression analysis of counts per million (cpm) normalized count data. Sequences with a perfect match or one mismatch were retained for further analysis. Differential miRNA expression profiling included fold difference estimates between different treatment and time course comparisons as well as $P$-values and false discovery rate (FDR) determinations for each differentially expressed species. Target identification of genes putatively regulated by miRNAs was performed using TargetScan [69]. Heatmaps were generated for differentially expressed miRNAs in each set of comparisons using R. Visualization of overlapping expression among miRNAs identified at different infection timepoints was done using Venny 2.0, an interactive Venn diagram tool for comparing name lists of up to 4 genomic datasets [70]. Software parameters and tools can be found in the Additional file 1.

Pathway, Core and Comparison analyses for all the FDR corrected differentially expressed miRNAs irrespective of time points in infected and reinfected mice were analyzed using the Ingenuity Pathway Analysis software (Qiagen 
Inc.) and Daina MirPath [30]. The top networks, molecular and cellular functions, diseases and disorders and physiological system development and functions were determined using Ingenuity System Interactive Pathway Systems (version $18,488,943$ ). Stringency was set at 'highly predicted' and 'experimentally validated'. The software uses its own internal algorithm and other databases, including TarBase, TargetScan and miRecords, as well as findings published in the literature. Complete reference set for this analysis was carried out using the Ingenuity Knowledge Base (Genes+ Endogenous Chemicals) for all molecules associated with diseases, functions, pathway, or list annotations.

This study focused on the top networks, molecular and cellular functions, diseases and disorders and physiological system development and functions for each infection. Confidence was set at 'moderately predicted', 'highly predicted' and 'experimentally validated. A threshold value of $-\log$ ( $p$-value) between 1.33 and 21.45 was set to identify molecules whose expression was significantly differentially regulated. The right-tailed Fisher's exact test was used to calculate a p-value determining the probability that each biological function and/or disease assigned to that data set is due to chance alone [71]. IPA library of canonical pathways that were most significant to the infected and reinfected mice microRNA data set were carried out. Comparison analysis, which analyzed the changes in miRNA expression across observations recorded, was also performed. Graphical representation of the networks generated using molecular relationships between molecules and miRNAs expressed were generated using the IPA software. Molecules are represented as nodes, and the biological relationship between two nodes is represented as an edge (line). All edges are supported by at least one reference from the literature, from a textbook, or from canonical information stored in the Ingenuity Pathways Knowledge Base. Nodes are displayed using various shapes that represent the functional class of the gene product.

\section{MiRNA validation}

Quantitative real time (qRT)-PCR was performed to validate differentially-expressed miRNAs discovered through the deep sequencing. cDNA synthesis and qRT-PCR was performed using Exiqon qPCR microRNA assays kits (Exiqon, Woburn, MA) per the manufacturer's protocol. The cycle threshold $(\mathrm{Ct})$ values and the relative expression of miRNAs in infected and control samples were normalized to U6 miRNA expression. The qRT-PCR data was analyzed using the delta-delta- $\mathrm{Ct}(\mathrm{ddCT})$ method [10].

\section{Statistical analysis}

The data derived from non-sequencing experiments were analyzed and compared by performing a 1 - or 2-tailed t-test, and the relationship between different experimental groupings was assessed by analysis of variance (ANOVA) using GraphPad Prism 5.0 software (GraphPad Software, Inc., La Jolla, CA). Statistical significance was judged at $P<0.05$.

\section{Additional file}

\begin{abstract}
Additional file 1: Figure S1. Venn diagram of differentially expressed miRNAs after chlamydia infection. Figure S2. Venn diagram of differentially expressed miRNAs after chlamydia reinfection. Figure S3. Pathways predicted to be regulated by miRNAs differentially expressed in mice after chlamydia infection. Figure S4. Pathways predicted to be regulated by miRNAs differentially expressed in mice after chlamydia reinfection. Figure S5. A: Network One for Chlamydia infection shows CEBPA a transcription regulator as the focus molecule with associated miRNAs. B: Network Two for Chlam ydia infection shows CREB1 a transcription regulator as the focus molecule with associated miRNAs. C: Network One for Chlamydia reinfection shows RHOA, an enzyme, as the focus molecule with associated miRNAs and other molecules D: Network Two for Chlamydia reinfection shows mir-423-5p as the focus molecule with associated miRNAs and other molecules. E: Network Three for Chlamydia reinfection shows TNFSF10 as the focus molecule with associated miRNAs and other molecules. F: Network Four for Chlamydia reinfection shows the kinase SRC as the focus molecule with associated miRNAs and other molecules. G: Network Five for Chlamydia reinfection shows the transporter BCL2 as the focus molecule with associated miRNAs and other molecules. Table S1. Summary Analysis of Top Diseases and Biological Functions of miRNA expressed in the Chlamydia Infection and Re-Infection. Table S2. Networks for the Top Diseases and Biological Function Category of miRNA expressed in the Chlamydia Reinfection. Figure S6. Example of quality scoring for miRNA sequencing in this study. Figure S7. (A) Validation of miRNA 378b expression after single infection using $\mathrm{qPCR}$, (B) Validation of miRNA 142-5p expression after single infection using $q P C R$. Figure S8. Comprehensive Analysis Pipeline for miRNA-seq data (CAP-miRSeq), adapted from Sun et al., 2014 [35]. (PDF $1710 \mathrm{~kb}$ )
\end{abstract}

\section{Abbreviations}

AKT: Protein kinase B; ANOVA: Analysis of variance; BAM: Binary alignment map; CAP-miRSeq: Comprehensive analysis pipeline for miRNA-sequence data; cDNA: Complementary DNA; CEBPA: CCAAT/Enhancer Binding Protein Alpha; Cpm: Counts per million; CREB1: CAMP Responsive Element Binding Protein 1; Ct: Cycle threshold; ddCt: delta-delta- Ct; EBs: Elementary bodies; ECM: Extra cellular matrix; EMT: Epithelial to mesenchymal transition; FCUR: FCy receptor; FDR: False discovery rate; FIG: Figure; FNDC1: fibronectin type III domain containing 1; G1: Gap 1 stage; GEO: Gene Expression Omnibus; GGBC: Georgia Genomic and Bioinformatics Core; HE: Hematoxylin and eosin; HPV: herpes virus; IACUC: Institutional Animal Care and Use Committee; IFU: Inclusion-forming units; IPA: Ingenuity pathway analysis software; JAK2: Janus kinase 2; miRNA(s): MicroRNA(s); miRNA-seq: miRNAsequencing; MMP2: Matrix metallopeptidase 2; MSM: Morehouse School of Medicine; mTor: mammalian target of rapamycin; NCBI: National Center for Biotechnology Information; NGS: Next generation sequencing;

NICHD: National Institute of Child Health and Human Development; NIGMS: National Institute of General Medical Sciences; NIH: National Institute of Health; NIMHD: National Institute on Minority Health and Health Disparities; PERK: Protein kinase RNA-like endoplasmic reticulum kinase; PHS: Public Health Service; PI3: Phosphoinositide 3-kinase; PID: Pelvic inflammatory disease; qPCR: Quantitative PCR; qRT-PCR: Quantitative real time PCR; Rho GDI alpha: Rho GDP dissociation inhibitor alpha; RHOA: Ras Homolog Family Member A; RNA: Ribonucleic acid; S: Synthesis stage; SAM: Sequence alignment map; SP1: Specificity Protein 1; TFI: Tubal factor infertility; TGF: Transforming growth factor beta; TNFSF10: Tumor necrosis factor superfamily member 10

\section{Acknowledgements}

YO would like to thank Dr. Jessica Kissinger, Director of the Institute of Bioinformatics, University of Georgia, she has been a awesome mentor and 
was instrumental in making the resourses available at the University of Georgia.

We would like to extend our thanks to the Georgia Genomic and Bioinformatic Core for the assistance during the sequencing process, especially Drs. Magdy Aladaby and Myriam Belanger for working on designing the primer sequences for the sequencing and meeting up with the deadlines. I would also like to thank Mr. Roger Nilsen for his role in sequencing the miRNAs.

This manuscript is dedicated to Dr. Qing He. Dr. He who in the manuscript author contribution is denoted by Q.H, passed away on Saturday June 2nd 2018 .

\section{Funding}

This work was supported by NIH grant 8G12MD007602 from the National Institute on Minority Health and Health Disparities (NIMHD), 1SC1All03041-01A1 from National Institute of General Medical Sciences (NIGMS) to Q.H. and 1SC2HD086066-01A1 from Eunice Kennedy Shriver National Institute of Child Health and Human Development (NICHD) to Y.O. T.S is an MSM Research Initiative for Scientific Enhancement (RISE) Scholar. The funding agencies did not have a role in the design of the study and in the collection, analysis, and interpretation of data and in writing the manuscript.

\section{Availability of data and materials}

The authors have deposited the raw sequences and processed data in the National Center for Biotechnology Information (NCBI) Gene Expression Omnibus (Geo) webpage https://www.ncbi.nlm.nih.gov/geo/query/ acc.cgi?acc=GSE118396, and is available for download.

\section{Authors' contribution}

T.S, Y.W, K.R., D.M., S.L., Z.G., R.P., D.E., Q.H. and Y.O performed the experiments; Y.W, T.S, K.R., D.M., Q.H. and Y.O. performed the animal work; U.B., Q.H. and Y.O performed histological analysis; W.W.L., I.B., S.L., T.O., Q.H., and Y.O., assisted with data analysis; W.T., U.B., F.E., C.B., J.U.I. Q.H., and Y.O., contributed to the study design and I.B., W.W.L., T.O., U.B., F.E., C.B., J.U.I., Q.H and Y.O participated in the manuscript preparation. All authors read and approved the final manuscript.

\section{Competing interest}

The authors declare no competing interest, and that the funders had no role in study design, data collection and analysis, decision to publish, or preparation of the manuscript. The authors have deposited the raw sequences and processed data in the NCBI Geo webpage.

\section{Ethics approval and consent to participate}

The Institutional Animal Care and Use Committee of Morehouse School of Medicine (MSM-IACUC) approved the animal care and use protocol (\# 16-24), which we followed in this study.

\section{Consent for publication}

Not applicable.

\section{Publisher's Note}

Springer Nature remains neutral with regard to jurisdictional claims in published maps and institutional affiliations.

\section{Author details}

'Department of Microbiology, Biochemistry \& Immunology, Morehouse School of Medicine, 720 Westview Drive, S.W, Atlanta, GA 30310, USA. ${ }^{2}$ Centers for Disease Control \& Prevention (CDC), Atlanta, GA 30333, USA. ${ }^{3}$ Department of Pathology, University of Georgia, College of Veterinary Medicine, Athens, GA 30602, USA. ${ }^{4}$ Institute of Bioinformatics, University of Georgia, Athens, GA 30602, USA. 5Department of Physical Sciences, Georgia State University, Covington, GA 30014, USA. ${ }^{6}$ Department of Physiology, Morehouse School of Medicine, Atlanta, GA 30310, USA.

Received: 27 August 2018 Accepted: 29 January 2019 Published online: 18 February 2019

\section{References}

1. Cates W Jr, Wasserheit JN. Genital chlamydial infections: epidemiology and reproductive sequelae. Am J Obstet Gynecol. 1991;164(6 Pt 2):1771-81.
2. Darville T, Hiltke TJ. Pathogenesis of genital tract disease due to chlamydia trachomatis. J Infect Dis. 2010;201(Suppl 2):S114-25.

3. Soper DE. Pelvic inflammatory disease. Obstet Gynecol. 2010;116(2 Pt 1): 419-28.

4. Workowski KA, Bolan GA, Centers for Disease C. Prevention. Sexually transmitted diseases treatment guidelines, 2015. MMWR Recomm Rep. 2015; 64(RR-03):1-137.

5. Park ST, Lee SW, Kim MJ, Kang YM, Moon HM, Rhim CC. Clinical characteristics of genital chlamydia infection in pelvic inflammatory disease. BMC Womens Health. 2017;17(1):5.

6. Bastidas RJ, Elwell CA, Engel JN, Valdivia RH. Chlamydial intracellular survival strategies. Cold Spring Harb Perspect Med. 2013;3(5):a010256.

7. Lewis ME, Belland RJ, AbdelRahman YM, Beatty WL, Aiyar AA, Zea AH, et al. Morphologic and molecular evaluation of chlamydia trachomatis growth in human endocervix reveals distinct growth patterns. Front Cell Infect Microbiol. 2014;4:71.

8. O'Connell CM, Ferone ME. Chlamydia trachomatis genital infections. Microb Cell. 2016;3(9):390-403.

9. Ficarra M, Ibana JS, Poretta C, Ma L, Myers L, Taylor SN, et al. A distinct cellular profile is seen in the human endocervix during chlamydia trachomatis infection. Am J Reprod Immunol. 2008;60(5):415-25.

10. Igietseme JU, Omosun Y, Partin J, Goldstein J, He Q, Joseph K, et al. Prevention of chlamydia-induced infertility by inhibition of local caspase activity. J Infect Dis. 2013;207(7):1095-104.

11. Omosun Y, McKeithen D, Ryans K, Kibakaya C, Blas-Machado U, Li D, et al. Interleukin-10 modulates antigen presentation by dendritic cells through regulation of NLRP3 inflammasome assembly during chlamydia infection. Infect Immun. 2015;83(12):4662-72.

12. Prozialeck WC, Fay MJ, Lamar PC, Pearson CA, Sigar I, Ramsey KH. Chlamydia trachomatis disrupts $\mathrm{N}$-cadherin-dependent cell-cell junctions and sequesters beta-catenin in human cervical epithelial cells. Infect Immun. 2002;70(5):2605-13.

13. Igietseme JU, Omosun Y, Stuchlik O, Reed MS, Partin J, He Q, et al. Role of epithelial-mesenchyme transition in chlamydia pathogenesis. PLoS One. 2015;10(12):e0145198.

14. Stallmann S, Hegemann JH. The chlamydia trachomatis Ctad1 invasin exploits the human integrin beta1 receptor for host cell entry. Cell Microbiol. 2016:18(5):761-75.

15. Kari L, Southern TR, Downey CJ, Watkins HS, Randall LB, Taylor LD, et al. Chlamydia trachomatis polymorphic membrane protein $D$ is a virulence factor involved in early host-cell interactions. Infect Immun. 2014;82(7): 2756-62.

16. Weber MM, Lam JL, Dooley CA, Noriea NF, Hansen BT, Hoyt FH, et al. Absence of specific chlamydia trachomatis inclusion membrane proteins triggers premature inclusion membrane lysis and host cell death. Cell Rep. 2017;19(7):1406-17.

17. Yeruva L, Myers GS, Spencer N, Creasy HH, Adams NE, Maurelli AT, et al. Early microRNA expression profile as a prognostic biomarker for the development of pelvic inflammatory disease in a mouse model of chlamydial genital infection. MBio. 2014;5(3):e01241-14.

18. Gupta R, Arkatkar T, Yu JJ, Wali S, Haskins WE, Chambers JP, et al. Chlamydia muridarum infection associated host MicroRNAs in the murine genital tract and contribution to generation of host immune response. Am J Reprod Immunol. 2015;73(2):126-40.

19. Dugas DV, Bartel B. MicroRNA regulation of gene expression in plants. Curr Opin Plant Biol. 2004;7(5):512-20.

20. Yekta S, Shih $\mathrm{H}$, Bartel DP. MicroRNA-directed cleavage of HOXB8 mRNA. Science. 2004;304(5670):594-6.

21. Shyu $A B$, Wilkinson MF, van Hoof $A$. Messenger RNA regulation: to translate or to degrade. EMBO J. 2008;27(3):471-81.

22. Derrick T, Roberts C, Rajasekhar M, Burr SE, Joof H, Makalo P, et al. Conjunctival MicroRNA expression in inflammatory trachomatous scarring. PLoS Negl Trop Dis. 2013;7(3):e2117.

23. Derrick T, Last AR, Burr SE, Roberts $\mathrm{CH}$, Nabicassa M, Cassama E, et al. Inverse relationship between microRNA-155 and -184 expression with increasing conjunctival inflammation during ocular chlamydia trachomatis infection. BMC Infect Dis. 2016;16:60.

24. Gupta R, Arkatkar T, Keck J, Koundinya GK, Castillo K, Hobel S, et al. Antigen specific immune response in chlamydia muridarum genital infection is dependent on murine microRNAs-155 and -182. Oncotarget. 2016;7(40): $64726-42$. 
25. Yeruva L, Pouncey DL, Eledge MR, Bhattacharya S, Luo C, Weatherford EW, et al. MicroRNAs Modulate Pathogenesis Resulting from Chlamydial Infection in Mice. Infect Immun. 2017:85(1). https://doi.org/10.1128/IAl.00768-16.

26. Chowdhury SR, Reimer A, Sharan M, Kozjak-Pavlovic V, Eulalio A, Prusty $B K$, et al. Chlamydia preserves the mitochondrial network necessary for replication via microRNA-dependent inhibition of fission. J Cell Biol. 2017;216(4):1071-89.

27. De Clercq E, Kalmar I, Vanrompay D. Animal models for studying female genital tract infection with chlamydia trachomatis. Infect Immun. 2013:81(9):3060-7.

28. de la Maza LM, Pal S, Khamesipour A, Peterson EM. Intravaginal inoculation of mice with the chlamydia trachomatis mouse pneumonitis biovar results in infertility. Infect Immun. 1994;62(5):2094-7.

29. Barron AL, White HJ, Rank RG, Soloff BL, Moses EB. A new animal model for the study of chlamydia trachomatis genital infections: infection of mice with the agent of mouse pneumonitis. J Infect Dis. 1981;143(1):63-6.

30. Vlachos IS, Zagganas K, Paraskevopoulou MD, Georgakilas G, Karagkouni D, Vergoulis T, et al. DIANA-miRPath v3.0: deciphering microRNA function with experimental support. Nucleic Acids Res. 2015;43(W1):W460-6.

31. Arkatkar T, Gupta R, Li W, Yu JJ, Wali S, Neal Guentzel M, et al. Murine MicroRNA214 regulates intracellular adhesion molecule (ICAM1) gene expression in genital chlamydia muridarum infection. Immunology. 2015;145(4):534-42.

32. Hillis SD, Owens LM, Marchbanks PA, Amsterdam LF, Mac Kenzie WR. Recurrent chlamydial infections increase the risks of hospitalization for ectopic pregnancy and pelvic inflammatory disease. Am J Obstet Gynecol. 1997;176(1 Pt 1):103-7.

33. Eminaga S, Christodoulou DC, Vigneault F, Church GM, Seidman JG. Quantification of microRNA expression with next-generation sequencing. Curr Protoc Mol Biol. 2013;Chapter 4:Unit 417

34. Pritchard CC, Cheng HH, Tewari M. MicroRNA profiling: approaches and considerations. Nat Rev Genet. 2012;13(5):358-69.

35. Sun Z, Evans J, Bhagwate A, Middha S, Bockol M, Yan H, et al. CAPmiRSeq: a comprehensive analysis pipeline for microRNA sequencing data. BMC Genomics. 2014;15:423.

36. Igietseme JU, Omosun Y, Nagy T, Stuchlik O, Reed MS, He Q, et al. Molecular Pathogenesis of Chlamydia Disease Complications: Epithelial-Mesenchymal Transition and Fibrosis. Infect Immun. 2018;86(1). https://doi.org/10.1128/IAI. 00585-17.

37. Morrison RP, Caldwell HD. Immunity to murine chlamydial genital infection. Infect Immun. 2002;70(6):2741-51.

38. McGinnis LK, Luense $L$, Christenson LK. MicroRNA in ovarian biology and disease. Cold Spring Harb Perspect Med. 2015;5(9):a022962.

39. Chen QG, Zhou W, Han T, Du SQ, Li ZH, Zhang Z, et al. MiR-378 suppresses prostate cancer cell growth through downregulation of MAPK1 in vitro and in vivo. Tumour Biol. 2016;37(2):2095-103.

40. Donadeu FX, Schauer SN, Sontakke SD. Involvement of miRNAs in ovarian follicular and luteal development. J Endocrinol. 2012;215(3):323-34.

41. Hyun J, Wang S, Kim J, Rao KM, Park SY, Chung I, et al. MicroRNA-378 limits activation of hepatic stellate cells and liver fibrosis by suppressing Gli3 expression. Nat Commun. 2016;7:10993.

42. Wang B, Yao K, Wise AF, Lau R, Shen $H H$, Tesch $G H$, et al. miR-378 reduces mesangial hypertrophy and kidney tubular fibrosis via MAPK signalling. Clin Sci (Lond). 2017;131(5):411-23.

43. Wang XL, Zhang T, Wang J, Zhang DB, Zhao F, Lin XW, et al. MiR-378b Promotes Differentiation of Keratinocytes through NKX3.1. PLoS One. 2015; 10(8):e0136049.

44. Wang Z, Ma B, Ji X, Deng Y, Zhang T, Zhang X, et al. MicroRNA-378-5p suppresses cell proliferation and induces apoptosis in colorectal cancer cells by targeting BRAF. Cancer Cell Int. 2015;15:40.

45. Xu S, Linher-Melville K, Yang BB, Wu D, Li J. Micro-RNA378 (miR-378) regulates ovarian estradiol production by targeting aromatase. Endocrinology. 2011;152(10): 3941-51.

46. Luedde T. MicroRNA-151 and its hosting gene FAK (focal adhesion kinase) regulate tumor cell migration and spreading of hepatocellular carcinoma. Hepatology. 2010;52(3):1164-6.

47. Ding J, Huang S, Wu S, Zhao Y, Liang L, Yan M, et al. Gain of miR-151 on chromosome 8q24.3 facilitates tumour cell migration and spreading through downregulating RhoGDIA. Nat Cell Biol. 2010;12(4):390-9.

48. Kuang H, Han D, Xie J, Yan Y, Li J, Ge P. Profiling of differentially expressed microRNAs in premature ovarian failure in an animal model. Gynecol Endocrinol. 2014;30(1):57-61.
49. Liu Z, Gersbach E, Zhang X, Xu X, Dong R, Lee $P$, et al. miR-106a represses the $\mathrm{Rb}$ tumor suppressor p130 to regulate cellular proliferation and differentiation in high-grade serous ovarian carcinoma. Mol Cancer Res. 2013;11(11):1314-25.

50. Lv M, Zhang X, Jia H, Li D, Zhang B, Zhang H, et al. An oncogenic role of miR-142-3p in human T-cell acute lymphoblastic leukemia (T-ALL) by targeting glucocorticoid receptor-alpha and CAMP/PKA pathways. Leukemia. 2012;26(4):769-77.

51. Kee HJ, Park S, Kwon JS, Choe N, Ahn Y, Kook H, et al. B cell translocation gene, a direct target of miR-142-5p, inhibits vascular smooth muscle cell proliferation by down-regulating cell cycle progression. FEBS Lett. 2013; 587(15):2385-92

52. Qin B, Yang H, Xiao B. Role of microRNAs in endothelial inflammation and senescence. Mol Biol Rep. 2012;39(4):4509-18.

53. Hou J, Wang $P$, Lin L, Liu X, Ma F, An H, et al. MicroRNA-146a feedback inhibits RIG-I-dependent type I IFN production in macrophages by targeting TRAF6, IRAK1, and IRAK2. J Immunol. 2009;183(3):2150-8.

54. Gilabert-Estelles J, Braza-Boils A, Ramon LA, Zorio E, Medina P, Espana F, et al. Role of microRNAs in gynecological pathology. Curr Med Chem. 2012;19(15):2406-13.

55. Trabert B, Waterboer $T$, Idahl A, Brenner N, Brinton LA, Butt J, et al. Antibodies Against Chlamydia trachomatis and Ovarian Cancer Risk in Two Independent Populations. J Natl Cancer Inst. 2018. https://doi.org/ 10.1093/jnci/djy084. [Epub ahead of print]

56. Chen SJ, Wu P, Sun L, Zhou B, Niu W, Liu S, et al. miR-204 regulates epithelial-mesenchymal transition by targeting SP1 in the tubular epithelial cells after acute kidney injury induced by ischemia-reperfusion. Oncol Rep. 2017;37(2):1148-58.

57. Wang P, Lv HY, Zhou DM, Zhang EN. miR-204 suppresses non-small-cell lung carcinoma (NSCLC) invasion and migration by targeting JAK2. Genet Mol Res. 2016;15(2). https://doi.org/10.4238/gmr.15026415.

58. Xu G, Chen J, Jing G, Grayson TB, Shalev A. miR-204 targets PERK and regulates UPR signaling and beta-cell apoptosis. Mol Endocrinol. 2016; 30(8):917-24.

59. George Z, Omosun Y, Azenabor AA, Partin J, Joseph K, Ellerson D, et al. The roles of unfolded protein response pathways in chlamydia pathogenesis. J Infect Dis. 2017;215(3):456-65.

60. He QY, Wang GC, Zhang H, Tong DK, Ding C, Liu K, et al. miR-106a-5p suppresses the proliferation, migration, and invasion of osteosarcoma cells by targeting HMGA2. DNA Cell Biol. 2016;35(9):506-20.

61. Wu J, Huang J, Wang W, Xu J, Yin M, Cheng $N$, et al. Long non-coding RNA Fer-1-like protein 4 acts as a tumor suppressor via miR-106a-5p and predicts good prognosis in hepatocellular carcinoma. Cancer Biomark. 2017;20(1):55-65

62. Gao F, Sun M, Gong Y, Wang H, Wang Y, Hou H. MicroRNA-195a-3p inhibits angiogenesis by targeting Mmp2 in murine mesenchymal stem cells. Mol Reprod Dev. 2016;83(5):413-23.

63. Andrew DW, Cochrane M, Schripsema JH, Ramsey KH, Dando SJ, O'Meara $\mathrm{CP}$, et al. The duration of chlamydia muridarum genital tract infection and associated chronic pathological changes are reduced in IL-17 knockout mice but protection is not increased further by immunization. PLoS One. 2013;8(9):e76664.

64. Zhou C, Shen L, Mao L, Wang B, Li Y, Yu H. miR-92a is upregulated in cervical cancer and promotes cell proliferation and invasion by targeting FBXW7. Biochem Biophys Res Commun. 2015;458(1):63-9.

65. Humphrys MS, Creasy T, Sun Y, Shetty AC, Chibucos MC, Drabek EF, et al. Simultaneous transcriptional profiling of bacteria and their host cells. PLoS One. 2013;8(12):e80597.

66. Wang Z, Liu M, Zhu H, Zhang W, He S, Hu C, et al. miR-106a is frequently upregulated in gastric cancer and inhibits the extrinsic apoptotic pathway by targeting FAS. Mol Carcinog. 2013;52(8):634-46.

67. Igietseme JU, He Q, Joseph K, Eko FO, Lyn D, Ananaba G, et al. Role of T lymphocytes in the pathogenesis of chlamydia disease. J Infect Dis. 2009; 200(6):926-34.

68. Bioinformatics B. FastQC-A quality control tool for high throughput sequence data. 2016. Available from: FastQCC Babraham Bioinformatics http://www.bioinformatics.babraham.ac.uk/projects/fastqc/.

69. Agarwal V, Bell GW, Nam JW, Bartel DP. Predicting effective microRNA target sites in mammalian mRNAs. elife. 2015;4. https://doi.org/10.7554/eLife.05005.

70. Oliveros JC. Venny. An interactive tool for comparing lists with Venn's diagrams. 2007-20015. Available from: http://bioinfogp.cnb.csic.es/tools/venny/index.html.

71. Kramer A, Green J, Pollard J Jr, Tugendreich S. Causal analysis approaches in ingenuity pathway analysis. Bioinformatics. 2014;30(4):523-30. 